Supporting Information for:

\title{
Laser Direct Write Synthesis of Lead Halide Perovskites
}

\author{
Stanley S. Chou ${ }^{\dagger, *}$, Brian S. Swartzentruber ${ }^{\ddagger}$, Matthew T. Janish ${ }^{\ddagger,}$, Kristin C. Meyer\|, \\ Laura B. Biedermann ${ }^{\dagger}$, Serdal Okur ${ }^{\perp}$, D. Bruce Burckel ${ }^{\dagger}$, C. Barry Carter $^{\star \S}$ and Bryan Kaehr\|,",** \\ ${ }^{\dagger}$ Sandia National Laboratories, Albuquerque, NM 87185, United States, United States; ${ }^{\star}$ Center for Integrated \\ Nanotechnologies, Sandia National Laboratories, Albuquerque, NM 87545, United States; ${ }^{\S}$ Department of Materials \\ Science \& Engineering, University of Connecticut, CT 06269, United States; "Advanced Materials Laboratory, \\ Sandia National Laboratories, Albuquerque, New Mexico 87106, United States; ${ }^{\perp}$ Department of Electrical and \\ Computer Engineering, the University of New Mexico, Albuquerque, New Mexico 87131, United States;

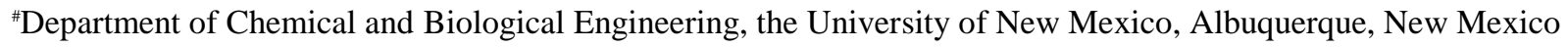 \\ 87131, United States \\ "correspondence to: schou@sandia.gov; bjkaehr@sandia.gov
}




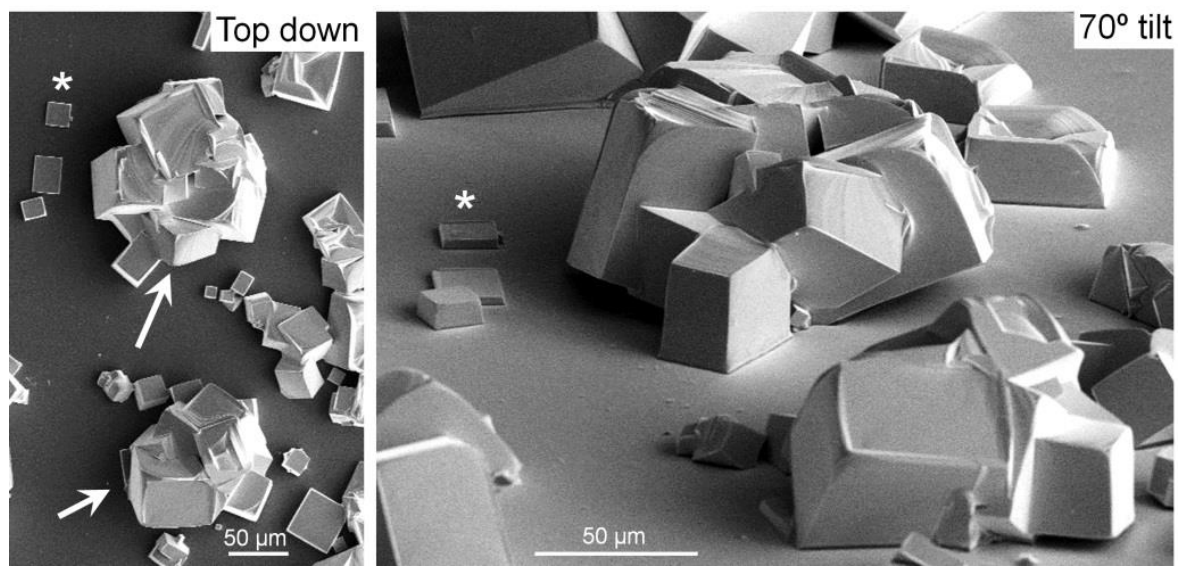

Figure S1. Top down and tilt views of scanning electron microscopy (SEM) images of two $\mathrm{CH}_{3} \mathrm{NH}_{3} \mathrm{PbBr}_{3}$ structures grown on platinum (arrows). Crystals surrounding these two structures are the result of solution precipitation during drying. The asterisk shows a visual reference point for orienting the two images.

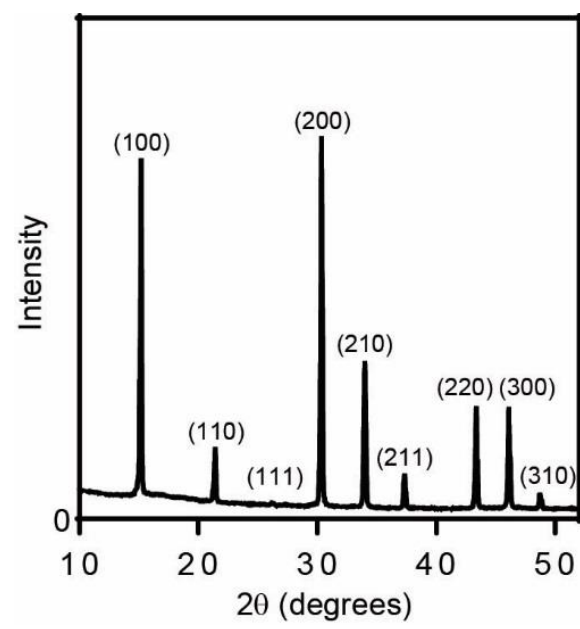

\begin{tabular}{ll}
\hline \hline Crystal System & cubic \\
Space group & $P$ m $3 m$ \\
$\mathbf{a}(\AA)$ & 5.83 \\
\hline
\end{tabular}

Figure S2. X-ray diffractogram of the $\mathrm{CH}_{3} \mathrm{NH}_{3} \mathrm{PbBr}_{3}$ materials examined in this work. 

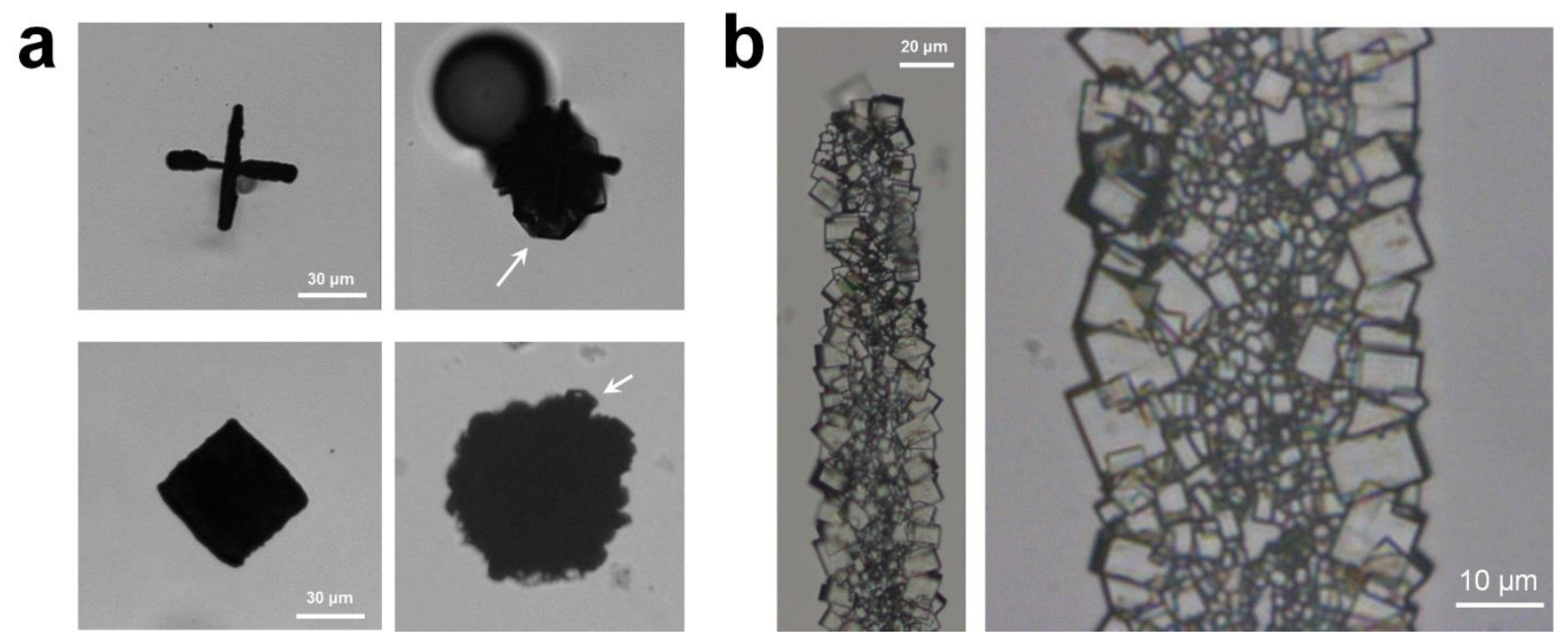

Figure S3. (a) Laser-induced fabrication of $\mathrm{CH}_{3} \mathrm{NH}_{3} \mathrm{PbI}_{3}$ perovskite on platinum (left panels) forms opaque, multi-crystallite structures (right panels are snap-shots of structures undergoing heat-induced crystallization) displaying crystal habits indicative of rhombo-hexagonal dodecahedrons (arrows). (b) Optical images of a laser direct write line of $\mathrm{CH}_{3} \mathrm{NH}_{3} \mathrm{PbCl}_{3}$ fabricated on sputtered carbon-functionalized coverglass shows the cuboid structure and optical transparency indicative of $\mathrm{CH}_{3} \mathrm{NH}_{3} \mathrm{PbCl}_{3}$ perovskites. 
a
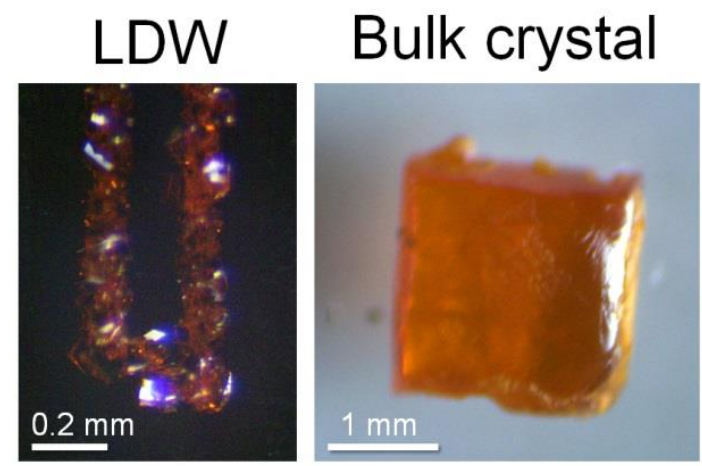

b

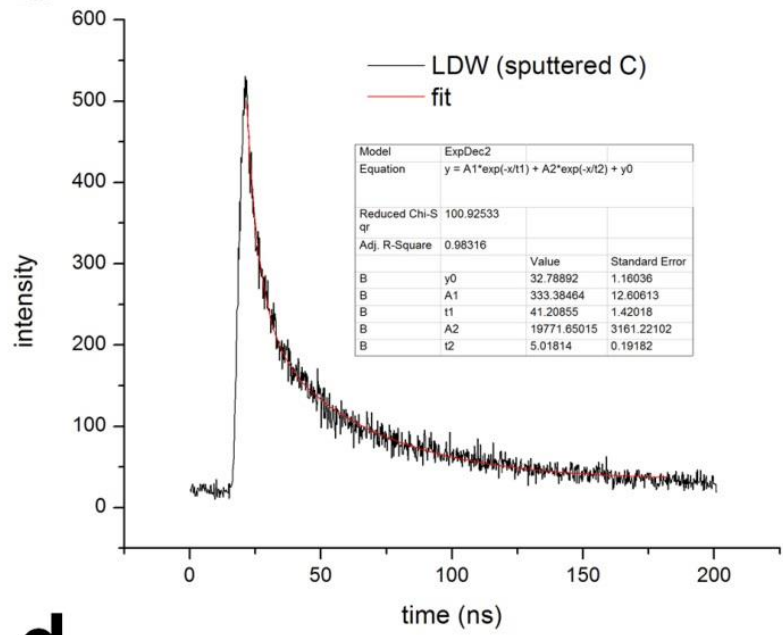

C

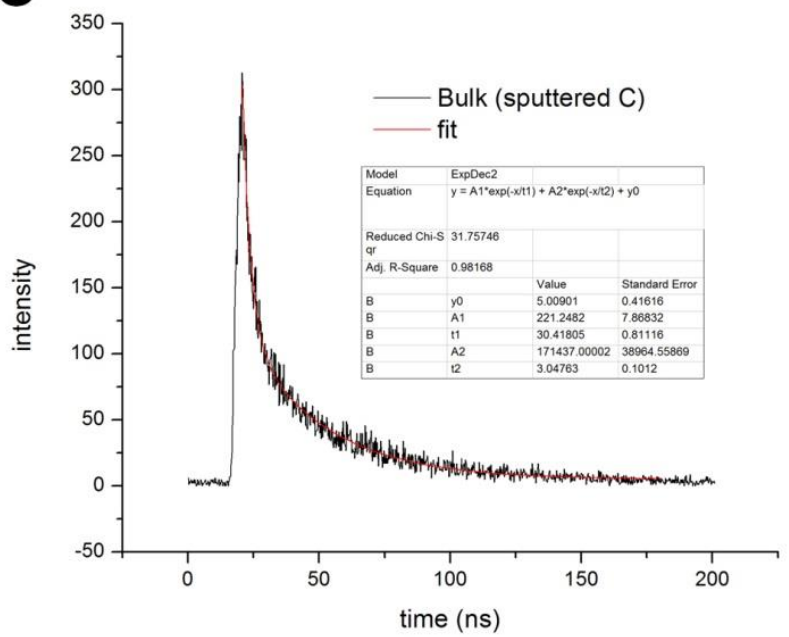

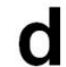

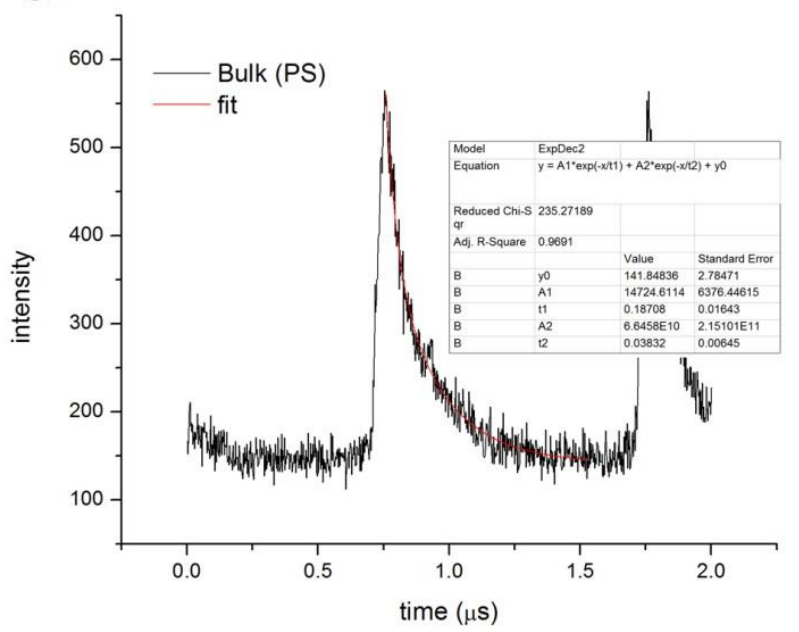

Figure S4. (a) Optical images of the $\mathrm{CH}_{3} \mathrm{NH}_{3} \mathrm{PbBr}_{3}$ materials (laser direct write, 'LDW', multicrystalline line on sputtered carbon and bulk solution grown single crystal, 'Bulk') examined using time resolved photoluminescence. Individual decay data and fitting of the photoluminescent decay curves combined in Figure 2 (panel f) for (b) laser direct write $\mathrm{CH}_{3} \mathrm{NH}_{3} \mathrm{PbBr}_{3}$ on sputtered carbon (c), bulk $\mathrm{CH}_{3} \mathrm{NH}_{3} \mathrm{PbBr}_{3}$ on sputtered carbon, and (d) the same $\mathrm{CH}_{3} \mathrm{NH}_{3} \mathrm{PbBr}_{3}$ crystal measured on an electrically insulating surface (polystyrene, PS). 

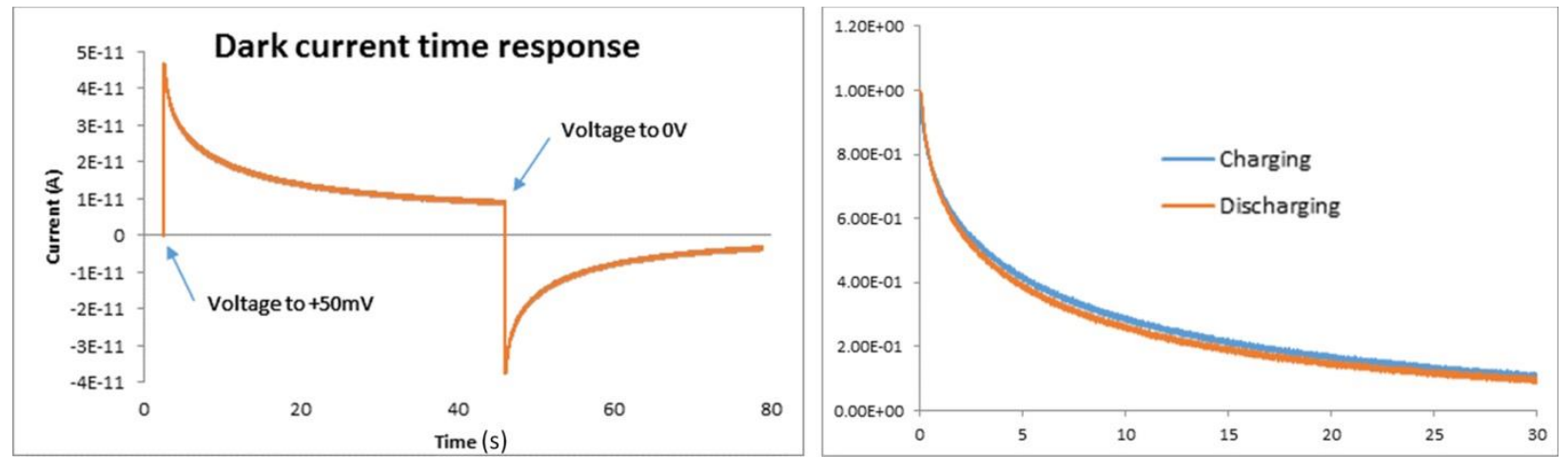

Figure S5. (left panel) Plot showing the dark current charge and discharge over time. The charge and discharge are nearly identical (right panel shows normalized curves) and can be fit by a stretched exponential function, $I(t)=e^{(-t / \tau)^{\beta}}$. The best fit for these data yield $\tau=2.49 \mathrm{~s}$ and $\beta$ $=0.54$. 